\title{
Relación entre la formación disciplinar y el ciclo profesional en el desarrollo de las habilidades blandas
}

\author{
Luis M. Moreno-Murcia y Yudy A. Quintero-Pulgar \\ Facultad de Ciencias Humanas y Sociales, Unidad de Investigación-Programa de Psicología, Corporación Universitaria \\ Minuto de Dios - UNIMINUTO. Bogotá-Colombia. (correo-e: luis.moreno.mu@uniminuto.edu.co; \\ yaquinterop@unal.edu.co)
}

Recibido Oct. 27, 2020; Aceptado Dic. 29, 2020; Versión final Feb. 18, 2021, Publicado Jun. 2021

\begin{abstract}
Resumen
Los objetivos de esta investigación son comparar el desarrollo de habilidades blandas en diferentes momentos del proceso formativo e identificar la relación entre la etapa de formación y la adquisición de habilidades en estudiantes de tres programas universitarios. La formación de habilidades blandas es relevante en escenarios académicos y laborales. Se utilizó un diseño descriptivo-correlacional de corte transversal; la muestra estuvo conformada por 116 estudiantes. Para determinar las habilidades blandas se aplicó la prueba CompeTEA y un ejercicio de observación. Los resultados indican que no existen diferencias estadísticamente significativas en habilidades blandas en relación con el ciclo profesional y la formación disciplinar. Se concluye que es necesario realizar un análisis crítico al interior de las instituciones educativas, que favorezca la armonización curricular o el diseño de programas de fortalecimiento y desarrollo de habilidades blandas, que en últimas da cuenta de la necesidad de una reforma al sistema educativo para una formación integral.
\end{abstract}

Palabras clave: habilidades blandas; educación superior: empleabilidad; revolución industrial

\section{Relationship between subject degree training and academic degree cycle in soft skills development}

\begin{abstract}
The objectives of this research study are to compare the development of soft skills at different points of student training and to determine the relationship between student training stage and skill acquisition in three university degree programs. A descriptive-correlational cross-sectional design is used. The sample is composed of 116 students. To determine soft skills, the CompeTEA test and an observational exercise are used. The results indicate that there are no statistically significant differences in soft skills in relation to academic degree cycle or degree program. It is concluded that it is necessary to perform critical analyses inside educational institutions to favor degree program curricular harmonization and design for strengthening and improving soft skills development. This highlights the need to reform educational systems to achieve a more comprehensive education.
\end{abstract}

Keywords: soft skills; higher education; employability; industrial revolution 


\section{INTRODUCCIÓN}

Las habilidades blandas también denominadas habilidades no cognitivas, habilidades socioemocionales, habilidades relacionales, competencias nucleares, habilidades transversales, habilidades genéricas 0 competencias para la empleabilidad, son todas aquellas capacidades adaptativas, cognitivas y comportamentales, que les permiten a las personas desenvolverse en contextos sociales, culturales e históricos, respondiendo a las necesidades y demandas de estos. (Ortega et al., 2016). Existen múltiples clasificaciones, en las que se destacan habilidades como: trabajo en equipo, liderazgo, pensamiento crítico, empatía, comunicación asertiva, motivación, manejo del tiempo, resiliencia, atención al detalle, adaptabilidad, resolución de problemas, autoconfianza, escucha activa, organización, entre otras (Cobo y Moravec, 2011; Robles, 2012).

En los últimos años, han despertado el interés de académicos, docentes, directivos e investigadores de diferentes instituciones de educación superior, debido al impacto que genera en el desarrollo individual, relacional, sociocultural y laboral en las sociedades modernas. Las investigaciones se han centrado en diferentes aspectos, entre los que se destacan: críticas al modelo educativo en el desarrollo de habilidades blandas (Buxarrais, 2013); articulación entre formación académica, habilidades blandas y diseño curricular (Keng, 2020; Tseng et al., 2019); importancia de las habilidades blandas en el mundo industrializado (Tito y Serrano, 2016); cuarta revolución industrial, empleabilidad y habilidades blandas (Bakhshi, et al., 2017; Davies et al., 2011); estrategias de formación en habilidades blandas (Ginting et al., 2020; Hariti y Rejeki, 2020); identificación de habilidades blandas (Rueda y Portilla, 2019; Wilson et al., 2014); relación entre empleabilidad, sector productivo y habilidades blandas (Araya y González, 2019; Dubey y Tiwarib,2020); entre otras.

Ahora bien, en la actualidad, las instituciones de educación superior enfrentan el desafío de formar a los estudiantes en lo teórico, disciplinar y práctico (competencias duras), pero también en habilidades transversales, cada día más requeridas en el mundo laboral y social, (Bakhshi et al., 2017; Dubey y Tiwarib, 2020; Schwab y Samans; 2016) atendiendo la formación integral del ser humano (Buxarrais, 2013; Moreno, 2019; Vera, 2016), que le permita tener un mejor desenvolvimiento en las diferentes áreas de acción, enfrentando, entre otras cuestiones, las desigualdades en términos oportunidades de acceso al conocimiento con las clases dominantes.

A pesar de ello, dichas instituciones se encuentran enfocadas principalmente en el desarrollo de competencias duras, desconociendo implícitamente el papel fundamental de las habilidades blandas. Según Guerra-Báez (2019) las competencias técnicas o "competencias duras" son necesarias para el ejercicio de una profesión y en la mayoría de los casos son el interés central de los centros universitarios, dado que las estrategias de evaluación de los aprendizajes adquiridos en la educación superior a nivel nacional e internacional, miden este tipo de competencias en los estudiantes y futuros egresados. Sin embargo, estudios recientes afirman que la adquisición de estas habilidades no necesariamente predice el éxito profesional, en cambio la formación integral si, donde se articulen habilidades blandas y duras; es allí donde se debe reconocer el papel fundamental de la universidad y su necesidad de transformación (Buxarrais, 2013; Dubey y Tiwarib, 2020; Tito y Serrano, 2016).

Para Ortega et al., (2016) es importante replantear el rol de la universidad en relación a la formación de habilidades blandas, ya sea mediante la reformulación total del sistema educativo, la armonización curricular - la capacitación y formación de docentes, con miras a implementar estrategias que garanticen un modelo integral (Dubey y Tiwarib, 2020; Musicco, 2018; Vera, 2016). Actualmente, la mayoría de programas académicos no cuentan con asignaturas específicas para este fin, asumiendo que las habilidades blandas son adquiridas en medio del proceso formativo. En consecuencia, las principales estrategias para fomentar el desarrollo de este tipo de competencias en el contexto universitario, se encuentran ligadas al currículo de forma implícita, articulando los conocimientos disciplinares a ejercicios prácticos o actividades experienciales, que impliquen procesos de interacción, relación, análisis, trabajo en equipo, comunicación, planeación, entre otros, relacionados con problemáticas reales, las cuales requieren en el estudiante, la puesta en marcha de habilidades duras y blandas al mismo tiempo (Ginting et al., 2020; Hariti y Rejeki, 2020; Ruiz, 2014).

Por ende, es importante analizar el desarrollo de estas habilidades en diferentes etapas del ciclo formativo, donde se evidencie si este, se da inherentemente o es necesaria la formulación de programas específicos, en los cuales se potencien y fortalezcan las habilidades blandas, independiente de la carrera y el ciclo profesional. Los objetivos de esta investigación son: comparar el desarrollo de habilidades blandas en diferentes momentos de la formación académica, asimismo, identificar la relación entre la adquisición de habilidades y diferentes etapas: inicio de la formación disciplinar, inicio de la práctica profesional y finalización de la práctica en estudiantes de tres programas académicos. Es conveniente resaltar que, en la literatura consultada, son nulos los estudios que intentan comprender dichas variaciones, teniendo como referente la profesión elegida y la etapa formativa, en la cual se encuentra el estudiante. 


\section{METODOLOGÍA}

La presente investigación se desarrolló bajo un enfoque cuantitativo de alcance descriptivo-correlacional de corte transversal. La muestra, no probabilística por conveniencia, estuvo conformada por 116 estudiantes universitarios de los cuales el $92 \%$ corresponden al género femenino, matriculados en tres programas de formación profesional, psicología, trabajo social y comunicación social, subdivididos en tres etapas diferentes de formación: inicio de la formación disciplinar, inicio de la práctica profesional y finalización del ciclo de prácticas.

En primer lugar, se convocó a los estudiantes a participar voluntariamente del estudio, se socializaron los objetivos y alcances, asimismo, se firmó el consentimiento informado. Posteriormente, se realizó un ejercicio interactivo basado en la teoría de Groh et al., (2015) con la finalidad de identificar conductas observables asociadas a diez habilidades blandas, se cambió la situación hipotética en la actividad, puesto que, en las primeras aplicaciones, los roles asignados eran confusos y algunos estudiantes no participaban por desconocimiento del rol. El ejercicio consistió en una discusión grupal, relacionada con la toma de decisiones, en la cual se proponía una situación que implica elegir 7 de 10 elementos de supervivencia, para arribar a una isla y sobrevivir 60 días. De acuerdo con esta situación hipotética, dos facilitadores evaluaron a cada participante: escucha activa, empatía, auto-confianza, comunicación asertiva, pensamiento crítico, liderazgo, cooperación, toma de decisiones, análisis y comprensión de las consecuencias, solución de problemas, trabajo en equipo, negociación, manejo emocional y análisis. El ejercicio tuvo una duración de 40 minutos.

También, se aplicó la prueba CompeTEA, un cuestionario diseñado por Arribas y Pereña (2015), que tiene como objetivo evaluar competencias a nivel personal y ocupacional, su diligenciamiento requiere aproximadamente 30 minutos. El test está compuesto por 170 ítems, divididos en 20 competencias que se encuentran agrupadas en 5 grandes áreas: área intrapersonal (autocontrol y estabilidad emocional, confianza en sí mismo y resistencia a la adversidad), área interpersonal (comunicación, establecimiento de relaciones, negociación, influencia y trabajo en equipo), área de desarrollo de tareas (iniciativa, orientación a resultados, capacidad de análisis y toma de decisiones), área del entorno (conocimiento de la empresa, visión y anticipación, orientación al cliente, apertura e identificación con la empresa) y área gerencial (liderazgo, planificación y organización). Las puntuaciones por ítem oscilan en una escala tipo Likert de frecuencia, en la que se presenta una situación o el grado de acuerdo con una afirmación. Los resultados se presentan en tres modalidades: nivel de desarrollo de la competencia (1-4), puntaje directo y escala típica con media en 50 y desviación estándar en 20.

Es relevante mencionar, que se usó esta prueba debido a su fácil implementación en términos de duración, modelo de aplicación (lápiz y papel) y resultados esperados (medición solo de competencias, sin patrones de personalidad). Adicional, la prueba cuenta con un baremo válido para población colombiana y ha sido aplicada en diferentes países latinoamericanos, por ejemplo, Chile, Argentina, Uruguay, Perú, México, entre otros y países europeos como España. A la fecha no se encuentran pruebas psicométricas con estas características en el mercado.

Finalmente, se analizaron los resultados mediante Software Statistical Package for the Social Sciences (SPSS) versión 25. En primer lugar, se establecieron los análisis descriptivos. En segundo lugar, se identificó si las variables cumplían con supuestos para pruebas paramétricas mediante las pruebas de Levene para igualdad de varianzas y Kolmogorov Smirnov. En tanto los datos no se agruparon de acuerdo a una distribución normal, el contraste entre grupos se realizó mediante el test de Kruskal-Wallis, alternativa no paramétrica a la ANOVA. Por último, se analizó la relación entre variables, teniendo en cuenta el coeficiente de correlación de Spearman, este también es una alternativa no paramétrica al coeficiente de correlación de Pearson. El nivel de significancia utilizado para la interpretación de los resultados fue $p<0.05$.

\section{RESULTADOS}

Inicialmente, se presentan los análisis descriptivos, con la finalidad de caracterizar la población de estudio. Posteriormente, los análisis de la comparación de grupos, por carrera y etapa de formación. Para finalizar, resultados de correlaciones entre las variables.

\section{Descriptivos}

La muestra estuvo conformada por 116 estudiantes universitarios, los cuales hacían parte de tres programas de formación profesional, psicología (44), trabajo social (56) y comunicación social (16). La mayoría de los estudiantes se encontraban iniciando su proceso de formación (43), cursando de primero a quinto semestre académico. Otros, se encontraban desempeñándose en su lugar de prácticas profesionales (34), dependiendo de sus diferentes líneas de acción. Por último, participaron estudiantes que ya habían terminado su ciclo de prácticas (39) como se observa en la tabla 1. 
Tabla 1: Caracterización de la muestra por carrera y ciclo de formación.

\begin{tabular}{|l|c|c|c|c|}
\hline Programa & $\begin{array}{c}\text { Inicio de formación } \\
\text { profesional }\end{array}$ & $\begin{array}{c}\text { Inicio de la práctica } \\
\text { profesional }\end{array}$ & $\begin{array}{c}\text { Finalización de la práctica } \\
\text { profesional }\end{array}$ & Total \\
\hline Psicología & 12 & 8 & 24 & 44 \\
\hline Trabajo Social & 26 & 23 & 8 & 56 \\
\hline $\begin{array}{l}\text { Comunicación } \\
\text { Social }\end{array}$ & 5 & 3 & 39 & 16 \\
\hline Total & 43 & 34 & & 116 \\
\hline
\end{tabular}

Los resultados de las observaciones del ejercicio interactivo, evidenciados en la tabla 2, indican que en promedio los estudiantes presentaron mayor número de conductas asociadas a la habilidad escucha activa (4.16). Por otra parte, competencias como oralidad, autoconfianza, trabajo en equipo y liderazgo se encontraron en un rango promedio de 3.5. Es importante, que habilidades como empatía (2.48), cooperación (2.42) y organización (1.91), presentaron el menor número de conductas.

Tabla 2: Análisis del ejercicio interactivo mediante observación de jueces.

\begin{tabular}{|l|c|c|c|c|}
\hline Habilidad & $\begin{array}{c}\text { Media } \\
(x)\end{array}$ & $\begin{array}{c}\text { Mediana } \\
(\mathrm{Me})\end{array}$ & $\begin{array}{c}\text { Desviación } \\
(s)\end{array}$ & $\begin{array}{c}\text { Varianza } \\
\left(s^{2}\right)\end{array}$ \\
\hline Escucha Activa & 4.16 & 5.0 & 1.21 & 1.47 \\
\hline Empatía & 2.48 & 3.0 & 2.12 & 4.53 \\
\hline Oralidad & 3.52 & 4.0 & 1.62 & 2.63 \\
\hline Autoconfianza & 3.28 & 4.0 & 1.57 & 2.48 \\
\hline Influencia & 3.38 & 4.0 & 1.72 & 2.96 \\
\hline Liderazgo & 3.42 & 4.0 & 1.59 & 2.54 \\
\hline Cooperación & 2.42 & 2.0 & 1.61 & 2.59 \\
\hline Iniciativa & 2.89 & 3.0 & 1.60 & 2.57 \\
\hline Organización & 1.91 & 2.0 & 1.66 & 2.75 \\
\hline Trabajo en equipo & 3.61 & 4.0 & 1.50 & 2.25 \\
\hline
\end{tabular}

Por último, En la discriminación por puntaje de las habilidades observadas en el ejercicio interactivo, se puede observar que la mayoría de los estudiantes obtuvieron puntajes entre 3 y 5 , a excepción de habilidades como empatía, cooperación y organización, las cuales se encuentran en puntajes entre 0 y 2 (tabla 3).

Tabla 3: Análisis por puntaje de las habilidades

\begin{tabular}{|l|c|c|c|c|c|c|}
\hline Habilidad & Puntaje 0 & Puntaje 1 & Puntaje 2 & Puntaje 3 & Puntaje 4 & Puntaje 5 \\
\hline $\begin{array}{l}\text { Escucha } \\
\text { Activa }\end{array}$ & 1.7 & 3.4 & 4.3 & 15.5. & 17.2 & 57.8 \\
\hline Empatía & 35.3 & 6.9 & 4.3 & 7.8 & 19.0 & 26.7 \\
\hline Oralidad & 12.1 & 1.7 & 7.8 & 12.1 & 33.6 & 32.8 \\
\hline Autoconfianza & 8.6 & 8.6 & 10.3 & 16.4 & 31.0 & 25.0 \\
\hline Influencia & 8.6 & 9.5 & 14.7 & 10.3 & 16.4 & 40.5 \\
\hline Liderazgo & 8.6 & 4.3 & 12.1 & 23.3 & 14.7 & 37.1 \\
\hline Cooperación & 12.1 & 21.6 & 20.7 & 19.8 & 9.5 & 16.4 \\
\hline Iniciativa & 10.3 & 8.6 & 25.0 & 14.7 & 20.7 & 20.7 \\
\hline Organización & 26.7 & 22.4 & 14.7 & 12.9 & 15.5 & 7.8 \\
\hline $\begin{array}{l}\text { Trabajo en } \\
\text { equipo }\end{array}$ & 8.6 & 1.7 & 9.5 & 13.8 & 32.8 & 33.6 \\
\hline
\end{tabular}

En relación con la prueba CompeTEA, se destaca que la mayoría de los participantes en promedio se encuentra por debajo de la media en las cinco grandes áreas, obteniendo puntuaciones deficientes e insuficientes en términos de desarrollo de habilidades: S-Intrapersonal (24.14), S-Interpersonal (28.92), SDesarrollo de Tareas (28.48), S-Entorno (21.75) y S-Gerencial (27.80), como se describe en la tabla 4.

Tabla 4: Cinco grandes áreas de la Prueba CompeTEA

\begin{tabular}{|l|c|c|c|c|}
\hline Habilidad & $\begin{array}{c}\text { Media } \\
(\mathrm{x})\end{array}$ & $\begin{array}{c}\text { Mediana } \\
(\mathrm{Me})\end{array}$ & $\begin{array}{c}\text { Desviación } \\
(\mathrm{s})\end{array}$ & $\begin{array}{c}\text { Varianza } \\
\left(\mathrm{s}^{2}\right)\end{array}$ \\
\hline S-Intrapersonal & 24.14 & 20 & 15.12 & 228.81 \\
\hline S-Interpersonal & 28.92 & 25 & 14.39 & 207.13 \\
\hline $\begin{array}{l}\text { S-Desarrollo } \\
\text { de Tareas }\end{array}$ & 28.48 & 25 & 17.23 & 297.03 \\
\hline S-Entorno & 21.75 & 20 & 12.66 & 160.46 \\
\hline S-Gerencial & 27.80 & 25 & 14.19 & 201.62 \\
\hline
\end{tabular}


En la tabla 5, al igual que en el análisis del ejercicio interactivo, se puede evidenciar que la mayoría de los estudiantes obtuvieron puntajes estandarizados entre 0 y 40 , evidenciando un nivel de desarrollo deficiente e insuficiente. Sin embargo, en la categoría S-interpersonal y S-Desarrollo de Tareas, aunque en términos generales se encuentran bajas, el $20 \%$ de los participantes tiene una puntuación aceptable. Es importante destacar, que de acuerdo con los puntajes estandarizados solo dos estudiantes se ubican en el rango sobresaliente y ninguno en excelente en ninguna de las áreas evaluadas.

Tabla 5: Puntajes directos de la prueba CompeTEA

\begin{tabular}{|l|c|c|c|c|c|}
\hline Variable & $\begin{array}{c}\text { Puntajes } \\
0-20\end{array}$ & $\begin{array}{c}\text { Puntajes de } \\
21 \text { a } 40\end{array}$ & $\begin{array}{c}\text { Puntajes de } \\
41 \text { a } 60\end{array}$ & $\begin{array}{c}\text { Puntajes de } \\
61 \text { a } 80\end{array}$ & $\begin{array}{c}\text { Puntajes de } \\
80 \text { a } 100\end{array}$ \\
\hline S-Intrapersonal & 50.86 & 35.34 & 13.79 & 0 & 0 \\
\hline S-Interpersonal & 31.03 & 47.41 & 20.69 & 0.86 & 0 \\
\hline S-Desarrollo de Tareas & 40.52 & 38.79 & 18.10 & 2.59 & 0 \\
\hline S-Entorno & 59.48 & 32.76 & 6.90 & 0.86 & 0 \\
\hline S-Gerencial & 38.79 & 46.55 & 13.79 & 0.86 & 0 \\
\hline
\end{tabular}

\section{Comparación entre grupos}

Los datos se analizaron para identificar si la distribución de las variables cumplía con los supuestos para usar pruebas paramétricas. La prueba de igualdad de varianzas de Levene arrojó un resultado que indica que todas las distribuciones de las variables son homocedásticas; no obstante, los resultados de la prueba de Kolmogorov Smirnov no permiten asumir el ajuste a una distribución normal, como se observa en las tablas 6 y 7. Por lo tanto, para el contraste entre grupos se utilizó la prueba Kruskal-Wallis. Los datos se interpretan con base en un nivel de significancia $p<0.05$.

Tabla 6: Resultados de pruebas para identificar criterios de normalidad e igualdad de varianzas-variables observación

\begin{tabular}{|l|c|c|c|c|c|c|}
\hline & \multicolumn{2}{|c|}{ Kolmogorov Smirnov } & \multicolumn{2}{c|}{ Prueba de homogeneidad de varianzas de Levene } \\
\hline Habilidad & $\begin{array}{c}\text { Estadístico de } \\
\text { la prueba }\end{array}$ & $\begin{array}{c}\text { Significancia } \\
\text { bilateral }\end{array}$ & $\begin{array}{c}F \\
\text { Etapa } \\
\text { formación }\end{array}$ & $\begin{array}{c}\text { Sig.bilateral } \\
\text { Etapa } \\
\text { formación }\end{array}$ & $\begin{array}{c}F \\
\text { Carrera }\end{array}$ & $\begin{array}{c}\text { Sig.bilateral } \\
\text { Carrera }\end{array}$ \\
\hline Escucha Activa & 0.332 & $<0.000$ & 1,163 & 0,316 & 0,743 & 0,478 \\
\hline Empatía & 0.232 & $<0.000$ & 0,881 & 0,417 & 0,527 & 0,592 \\
\hline Oralidad & 0.281 & $<0.000$ & 4,591 & 0,012 & 8,113 & 0,001 \\
\hline Autoconfianza & 0.238 & $<0.000$ & 1,485 & 0,231 & 2,338 & 0,101 \\
\hline Influencia & 0.232 & $<0.000$ & 0,411 & 0,664 & 1,390 & 0,253 \\
\hline Liderazgo & 0.209 & $<0.000$ & 1,083 & 0,342 & 1,398 & 0,251 \\
\hline Cooperación & 0.148 & $<0.000$ & 1,822 & 0,166 & 3,263 & 0,042 \\
\hline Iniciativa & 0.170 & $<0.000$ & 3,951 & 0,022 & 0,439 & 0,645 \\
\hline Organización & 0.200 & $<0.000$ & 1,016 & 0,365 & 1,664 & 0,194 \\
\hline $\begin{array}{l}\text { Trabajo en } \\
\text { equipo }\end{array}$ & 0.266 & $<0.000$ & 1,864 & 0,160 & 0,265 & 0,768 \\
\hline
\end{tabular}

Tabla 7: Resultados de pruebas para identificar criterios de normalidad e igualdad de varianzas-variables observación

\begin{tabular}{|l|c|c|c|c|c|c|}
\hline & \multicolumn{3}{|c|}{ Kolmogorov Smirnov } & \multicolumn{3}{c|}{ Prueba de homogeneidad de varianzas de Levene } \\
\hline Variable & $\begin{array}{c}\text { Estadístico de } \\
\text { la prueba }\end{array}$ & $\begin{array}{c}\text { Significancia } \\
\text { bilateral }\end{array}$ & $\begin{array}{c}F \\
\text { Etapa } \\
\text { formaci } \\
\text { ón }\end{array}$ & $\begin{array}{c}\text { Sig.bilateral } \\
\text { Etapa formación }\end{array}$ & $\begin{array}{c}F \\
\text { Carrera }\end{array}$ & $\begin{array}{c}\text { Sig.bilateral } \\
\text { Carrera }\end{array}$ \\
\hline S-Intrapersonal & 0.124 & $<0.000$ & 0,709 & 0,494 & 1,725 & 0,183 \\
\hline S-Interpersonal & 0.125 & $<0.000$ & 2,072 & 0,131 & 1,597 & 0,207 \\
\hline $\begin{array}{l}\text { S-Desarrollo de } \\
\text { Tareas }\end{array}$ & 0.106 & 0.003 & 0,951 & 0,389 & 1,048 & 0,354 \\
\hline S-Entorno & 0.150 & $<0.000$ & 1,675 & 0,192 & 3,209 & 0,044 \\
\hline S-Gerencial & 0.156 & $<0.000$ & 0,262 & 0,770 & 0,977 & 0,380 \\
\hline
\end{tabular}

Para identificar si existen diferencias estadísticas entre los grupos relacionadas con la etapa de formación, se realizó la prueba de comparación de Kruskal-Wallis, los resultados obtenidos en esta prueba para las variables evaluadas mediante observación, se describen en la tabla 8. Los respectivos estadísticos de contraste indican que no hay diferencias estadísticamente significativas en el desarrollo de habilidades blandas para los grupos que inician la etapa de formación disciplinar, aquellos que inician prácticas profesionales y los que han finalizado dicha etapa. Resultados similares se encuentran en la tabla 9 para las habilidades evaluadas mediante la prueba CompeTEA. 
Tabla 8: Resultados de pruebas Kruskal-Wallis para comparación de grupos- variables de observación: Etapa de formación

\begin{tabular}{|l|c|c|c|c|c|}
\hline & \multicolumn{2}{|c|}{ Mediana } & \multicolumn{2}{c|}{ Prueba Kruskal-Wallis } \\
\hline Habilidad & $\begin{array}{c}\text { Etapa } \\
\text { formación 1 }\end{array}$ & Etapa formación 2 & Etapa formación 3 & H de Kruskal-Wallis & Sig. Asintótica \\
\hline Escucha Activa & 5.00 & 5.00 & 5.00 & 1.32 & 0.51 \\
\hline Empatía & 4.00 & 1.50 & 3.00 & 1.12 & 0.56 \\
\hline Oralidad & 4.00 & 4.00 & 4.00 & 0.88 & 0.64 \\
\hline Autoconfianza & 3.00 & 4.00 & 4.00 & 3.35 & 0.18 \\
\hline Influencia & 4.00 & 4.00 & 4.00 & 0.03 & 0.98 \\
\hline Liderazgo & 4.00 & 4.50 & 2.00 & 0.38 & 0.82 \\
\hline Cooperación & 3.00 & 2.00 & 2.00 & 1.60 & 0.44 \\
\hline Iniciativa & 3.00 & 4.00 & 1.00 & 1.84 & 0.39 \\
\hline Organización & 2.00 & 1.50 & 4.00 & 2.77 & 0.52 \\
\hline Trabajo en equipo & 4.00 & 4.00 & & 1.27 & 0.25 \\
\hline
\end{tabular}

Tabla 9: Resultados de pruebas Kruskal-Wallis para comparación de grupos- variables prueba CompeTEA: Etapa de formación

\begin{tabular}{|l|c|c|c|c|c|}
\hline & \multicolumn{3}{|c|}{ Mediana } & \multicolumn{2}{c|}{ Prueba Kruskal-Wallis } \\
\hline Variable & Etapa formación 1 & $\begin{array}{c}\text { Etapa } \\
\text { formación 2 }\end{array}$ & $\begin{array}{c}\text { Etapa } \\
\text { formación 3 }\end{array}$ & $\begin{array}{c}\text { H de Kruskal- } \\
\text { Wallis. }\end{array}$ & Sig. Asintótica \\
\hline S-Intrapersonal & 25 & 20 & 25 & 0.514 & 0.773 \\
\hline S-Interpersonal & 25 & 30 & 25 & 2.268 & 0.322 \\
\hline S-Desarrollo de Tareas & 30 & 27.50 & 25 & 1.446 & 0.485 \\
\hline S-Entorno & 20 & 20 & 20 & 0.797 & 0.671 \\
\hline S-Gerencial & 25 & 27.50 & 25 & 0.428 & 0.807 \\
\hline
\end{tabular}

De otro modo, se realizó comparación de los puntajes obtenidos en cada una de las variables para los programas de formación (tabla 10), los resultados de la prueba Kuskal-Wallis indican que no hay diferencia estadísticamente significativa con relación a la carrera cursada.

Tabla 10: Resultados de pruebas Kruskal-Wallis para comparación de grupos- variables de observación: Carrera

\begin{tabular}{|l|c|c|c|c|c|}
\hline & \multicolumn{3}{|c|}{ Mediana } & \multicolumn{2}{c|}{ Prueba Kruskal-Wallis } \\
\hline Habilidad & Psicología & $\begin{array}{c}\text { Trabajo } \\
\text { Social }\end{array}$ & $\begin{array}{c}\text { Comunicación } \\
\text { Social }\end{array}$ & $\begin{array}{c}\text { H de Kruskal- } \\
\text { Wallis }\end{array}$ & Sig. Astótica \\
\hline Escucha Activa & 5.00 & 5.00 & 4.00 & 3.81 & 0.14 \\
\hline Empatía & 3.00 & 3.00 & 3.00 & 0.74 & 0.68 \\
\hline Oralidad & 4.00 & 4.00 & 4.00 & 1.63 & 0.44 \\
\hline Autoconfianza & 4.00 & 4.00 & 4.00 & 0.32 & 0.85 \\
\hline Influencia & 4.00 & 4.00 & 4.50 & 2.12 & 0.34 \\
\hline Liderazgo & 3.00 & 4.00 & 5.00 & 4.96 & 0.08 \\
\hline Cooperación & 2.00 & 2.50 & 2.00 & 0.11 & 0.94 \\
\hline Iniciativa & 2.00 & 3.00 & 3.50 & 4.63 & 0.09 \\
\hline Organización & 1.00 & 2.00 & 2.50 & 1.73 & 0.42 \\
\hline Trabajo en equipo & 4.00 & 4.00 & 4.00 & 1.72 & 0.42 \\
\hline
\end{tabular}

Si bien las medianas para liderazgo e iniciativa presentan puntuaciones más altas para comunicación social, éstas no son estadísticamente significativas. Algo similar ocurre con los resultados presentados en la tabla 11, los cuales señalan una puntuación más alta para comunicación social en la Dimensión Entorno, las cuales tampoco son estadísticamente significativas.

Tabla 11: Resultados de pruebas Kruskal-Wallis para comparación de grupos- variables prueba CompeTEA: Carrera

\begin{tabular}{|l|c|c|c|c|c|}
\hline & \multicolumn{3}{|c|}{ Mediana } & \multicolumn{2}{c|}{ Prueba Kruskal-Wallis } \\
\hline Variable & Psicología & Trabajo Social & Comunicación Social & H de Kruskal-Wallis. & Sig. Asintótica \\
\hline S-Intrapersonal & 20 & 20 & 30 & 1.42 & 0.49 \\
\hline S-Interpersonal & 25 & 25 & 30 & 1.87 & 0.39 \\
\hline S-Desarrollo de Tareas & 25 & 25 & 40 & 2.65 & 0.26 \\
\hline S-Entorno & 20 & 20 & 22.50 & 3.80 & 0.14 \\
\hline S-Gerencial & 25 & 25 & 27.50 & 1.13 & 0.56 \\
\hline
\end{tabular}




\section{Correlaciones entre variables}

Se realizó prueba de correlación de Spearman para las variables evaluadas, los resultados indican que hay correlación entre aquellas variables que corresponden a la misma prueba; al observar la relación entre las variables de las dos pruebas se observa que estas no presentan correlaciones estadísticamente significativas, aunque se esperaban asociaciones altas entre las pruebas dado que ambas evalúan dimensiones del constructo habilidades blandas.

Se resalta que las variables liderazgo, influencia y cooperación presentan índices de correlación más altos con otras variables de la prueba de observación, con relación a las variables de la prueba CompeTEA se encuentran relaciones similares, es decir, que las competencias interpersonales son las que presentan correlaciones más altas con el resto de las competencias. Lo anterior permite identificar que las competencias interpersonales tienen una relación con mayor desempeño en las demás variables, como se evidencia en la tabla 12.

Tabla 12: Correlación de Spearman para las variables evaluadas.

\begin{tabular}{|c|c|c|c|c|c|c|c|c|c|c|c|c|c|c|c|}
\hline & 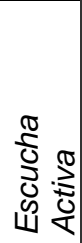 & 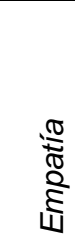 & 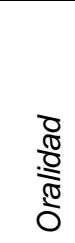 & 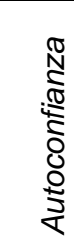 & $\frac{\frac{\pi}{d}}{\frac{\delta}{d}}$ & 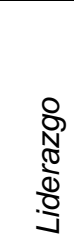 & 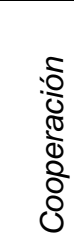 & 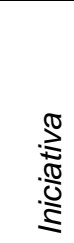 & 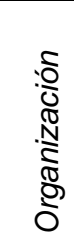 & 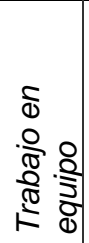 & 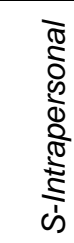 & $\begin{array}{l}\bar{d} \\
\delta \\
0 \\
\frac{d}{d} \\
\frac{2}{d} \\
\stackrel{D}{5} \\
\text { c' }\end{array}$ & 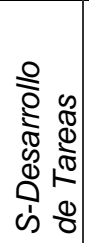 & 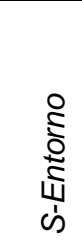 & 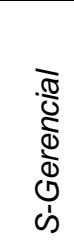 \\
\hline Escucha Activa & 1.00 & & & & & & & & & & & & & & \\
\hline Empatía & $.22^{* *}$ & 1.00 & & & & & & & & & & & & & \\
\hline Oralidad & $.33^{* *}$ & $.54^{* *}$ & 1.00 & & & & & & & & & & & & \\
\hline Autoconfianza & $.37^{* *}$ & $.61^{* *}$ & $.74^{* *}$ & 1.00 & & & & & & & & & & & \\
\hline Influencia & $29^{* *}$ & $.56^{* *}$ & $.60^{* *}$ & $.64^{* *}$ & 1.00 & & & & & & & & & & \\
\hline Liderazgo & $.25^{* *}$ & $.59^{* *}$ & $.63^{* *}$ & $.67^{* *}$ & $.78^{* *}$ & 1.00 & & & & & & & & & \\
\hline Cooperación & $.26^{* *}$ & $.55^{* *}$ & $.62^{* *}$ & $.68^{*+}$ & $.74^{* *}$ & $.76^{* *}$ & 1.00 & & & & & & & & \\
\hline Iniciativa & $.17^{*}$ & $.46^{* *}$ & $.47^{* *}$ & $.57^{* *}$ & $.77^{* *}$ & $.74^{* *}$ & $.71^{* *}$ & 1.00 & & & & & & & \\
\hline Organización & .09 & $.44^{* *}$ & $.48^{* *}$ & $.54^{* *}$ & $.67^{* *}$ & $.68^{* *}$ & $.68^{* *}$ & $.74^{* *}$ & 1.00 & & & & & & \\
\hline Trabajo en equipo & $.18^{*}$ & $.49^{* *}$ & $.49^{* *}$ & $.54^{* *}$ & $.72^{* *}$ & $.73^{* *}$ & $.66^{* *}$ & $69^{* *}$ & $.63^{* *}$ & 1.00 & & & & & \\
\hline S-Intrapersonal & .15 & $29^{* *}$ & $.22^{* *}$ & .15 & .12 & .13 & .11 & .07 & .02 & .09 & 1.00 & & & & \\
\hline S-Interpersonal & $.21^{*}$ & $.30^{* *}$ & $.19^{*}$ & $.23^{* *}$ & .14 & .12 & .05 & .12 & .07 & $.17^{*}$ & $.66^{* *}$ & 1.00 & & & \\
\hline S-Desarrollo de Tareas & .12 & $.20^{*}$ & .05 & .06 & .05 & .04 & .02 & .09 & .02 & .06 & $.59^{* *}$ & $.75^{* *}$ & 1.00 & & \\
\hline S-Entorno & .10 & $28^{* *}$ & .10 & .12 & .11 & .11 & .02 & .05 & .03 & .13 & $.62^{* *}$ & $.77^{* *}$ & $.71^{* *}$ & 1.00 & \\
\hline S-Gerencial & $.21^{*}$ & $24^{* *}$ & .14 & .15 & .13 & .11 & .10 & .11 & .04 & .12 & $.54^{* *}$ & $.76^{* *}$ & $.72^{* *}$ & $.68^{* *}$ & 1.00 \\
\hline
\end{tabular}

\section{DISCUSIÓN}

La comprensión de la evaluación, el desarrollo, el fortalecimiento y la puesta en práctica de las habilidades blandas es un tema de interés que se ha venido construyendo en las últimas décadas, debido al impacto directo que tienen en el desarrollo individual, relacional, socioeconómico, cultural, empresarial e industrial en la sociedad actual (Nickson, 2012). Las instituciones de educación superior han desempeñado un factor fundamental, puesto que se enmarcan dentro de los espacios de desarrollo integral del ser humano. Sin embargo, las críticas al sistema educativo se focalizan principalmente en la producción de conocimiento disciplinar con principios que se fundamentan en la industria y el capitalismo, promoviendo procesos de memorización, uniformidad, alineación, homogeneización, entre otros, desconociendo el papel fundamental de las habilidades blandas y asumiendo que estas se desarrollan inherentemente en medio del proceso de formación disciplinar (Buxarrais, 2013; Berry y Routon, 2020; Vera, 2016).

La presente investigación tuvo como objetivo comparar el desarrollo de habilidades blandas en diferentes momentos del proceso formativo, con la finalidad de conocer y evaluar su impacto en el desarrollo de habilidades blandas. Se encontró, que la mayoría de los estudiantes independientemente del ciclo y de la carrera presentan bajo niveles de habilidades blandas, esto puede relacionarse con los planteamientos de Buxarrais (2013), Ortega et al., (2016) y Vera (2016), referentes a la necesidad de las universidades en reformular su sistema educativo, realizar adaptaciones curriculares o promover programas de desarrollo de habilidades independientes a las asignaturas disciplinares (Keng, 2020; Tseng et al.,2019).

Igualmente, al encontrar bajos resultados, es importante cuestionar el rol de los docentes en este proceso. Estos cumplen un papel fundamental, puesto que son los primeros que deberían recibir formación y ser conscientes de la importancia y necesidad de dicho desarrollo, determinando su idoneidad a la hora promover espacios de fortalecimiento de habilidades blandas (López et al., 2017). En este orden de ideas, es necesario 
que los programas de desarrollo en competencias incorporen diferentes actores de la comunidad educativa, con la finalidad de alcanzar una formación integral, en directivos, docentes y estudiantes (Ngang et al., 2015; Rebele y Pierre; 2019).

De la misma forma, los puntajes bajos en habilidades blandas hallados en la muestra coinciden con la preocupación de los líderes del sector empresarial, puesto que la mayoría de los trabajadores no cuentan con las habilidades blandas para desenvolverse en el medio laboral y social, lo cual representa un reto en términos de las competencias de egreso para las instituciones de educación superior, estos déficit en formación afectan índices de empleabilidad, competitividad, desempeño y productividad. (Araya y González, 2019; Niño-Pinilla y Correa-Ocampo, 2019; Rueda y Portilla, 2019). Se evidencia que hay una diferencia entre los puntajes obtenidos por los estudiantes en la prueba escrita y los puntajes asignados por los facilitadores en el ejercicio de observación, a diferencia de otros estudios (Araya y González, 2019) para esta aplicación la percepción del estudiante frente a sus habilidades fue menor que la de observadores externos. Lo cual plantea un reto en términos metodológicos para identificar estrategias de evaluación de habilidades blandas (Zhang, 2012).

En cuanto a las limitaciones de la investigación, en primer lugar, se encuentra la utilización de instrumentos, aunque el ejercicio interactivo tuvo la validación por pares expertos, las observaciones pueden estar mediadas por significados, creencias y opiniones propias, afectando la validez y confiabilidad de los resultados. En segundo lugar, los puntajes objetivos en ambas pruebas, pueden estar relacionados con factores individuales que no se tuvieron en cuenta, por ejemplo: asistencia a programas de formación de habilidades, edad, nivel intelectual, ocupación, entre otros. En tercer lugar, el tamaño de la muestra fue reducido por áreas de conocimiento y periodos de formación. Para finalizar, es importante mencionar que el desarrollo, medición y caracterización de las habilidades blandas es un problema complejo y en permanente estudio (Ginting et al., 2020), puesto que, en términos individuales, cada habilidad tiene diferentes orígenes, posibilidades de desarrollo, fortalecimiento y aplicación, lo cual dificulta llevar a cabo investigaciones que abordan el concepto en general. Se sugiere para futuras investigaciones, realizar estudios de carácter psicométrico, con el objetivo de crear instrumentos o pruebas con estándares de confiabilidad y validez. Igualmente, estudios con muestras poblacionales mayores, variedad de áreas del conocimiento e inclusión de factores laborales.

\section{CONCLUSIONES}

A partir de los resultados obtenidos, se pueden extraer las siguientes conclusiones: 1. El nivel de habilidades blandas en estudiantes universitarios es bajo, se requiere, fortalecer el desarrollo de las mismas, ya sea mediante la reformulación curricular o cursos trasversales. Igualmente, da cuenta de la necesidad del sector educativo de realizar un análisis crítico frente a las necesidades de formación actuales asociadas a las competencias requeridas para la IV revolución industrial, asimismo, dicha reflexión debe incluir a diferentes sectores: empresariales, comunidades y estudiantes pues las competencias no solo deben orientarse al empleo sino a la formación para la vida. 2. Una etapa superior de la formación profesional no implica mayores puntajes en pruebas de habilidades blandas, estos puntajes pueden estas mediados por factores individuales, sociales, culturales, entre otros. 3. La reforma al sistema educativo es una necesidad de las sociedades modernas, para romper esquemas industrializados y capitalistas, mediante un mayor énfasis en el desarrollo del ser humano y el fortalecimiento de habilidades duras y blandas como modelo integral.

\section{REFERENCIAS}

Araya, E., y González, G., Propuesta para el fortalecimiento de habilidades técnicas, blandas y complementarias, y su impacto en el currículo TIC desde una perspectiva laboral, profesional y de gestión académica, http://dx.doi.org/10.22458/caes.v10i2.1907, Revista Electrónica Calidad en la Educación Superior, 10, (2), 112-14 (2019)

Bakhshi, H., Downing, J., Osborne, M., y Schneider, P., The future of skills: employment in 2030, Pearson and Nesta, London, Inglaterra (2017)

Belzunce, M., Danvila, I., y Martínez, F., Guía de competencias emocionales para directivos, 1를 Ed, 1-238, ESIC, Madrid, España, (2011)

Berry, R., y Routon, W., Soft skill change perceptions of accounting majors: current practitioner views versus their own reality, https://doi.org/10.1016/j.jaccedu.2020.100691, Journal of Accounting Education, 53, 100691 (2020)

Buxarrais, M., Nuevos valores para una nueva sociedad, un cambio de paradigma en educación, Edetania, (43), 53-65 (2013)

Cobo, C., y Moravec, J., Aprendizaje invisible hacia una nueva ecología de la educación 1-239, Col-lecció Transmedia XXI, Barcelona, España (2011)

Davies, A., Fidler, D., y Gorbis, M., Future work skills 2020., Institute for the Future (IFTF)., University of Phoenix Research Institute (2011) 
Dubey, R. y Tiwari, V., Operationalization of soft skill attributes and determining the existing gap in novice ICT professionals, https://doi.org/10.1016/j.ijinfomgt.2019.09.006, International Journal of Information Management, 50, 375386 (2020)

Guerra-Báez, S., Una revisión panorámica al entrenamiento de las habilidades blandas en estudiantes universitarios, http://dx.doi.org/10.1590/2175-35392019016464, Psicología Escolar y Educacional, 23, 1-11 (2019)

Ginting, H., Mahiranissa, A., Bekti, R., y Febriansyah, H., The effect of outing team building training on soft skills among MBA students, https://doi.org/10.1016/j.jime.2020.100423, The International Journal of Management Education,18(3), $100423(2020)$

Groh, M., Mckenzie, D., y Vishwanath, T., Reducing information asymmetries in the youth labor market of Jordan with psychometrics and skill based tests, https://doi.org/10.1093/wber/lhv005, The World Bank Economic Review, 29, 106$117(2015)$

Hariti, T., y Rejeki, S., Strengthening soft skills as the character of student nurses through the preceptorship management model, https://doi.org/10.1016/j.enfcli.2019.11.022, Enfermería Clínica, 30, 64-68 (2020)

Keng, L., The prceived importance of soft (service) skills in nursing care: a research study, Nurse Education Today, https://doi.org/10.1016/j.nedt.2019.104302, 85, 104302 (2020)

López-Mondéjar, L., y Tomás, L., Development of socioemotional skills through cooperative learning in a university environment, https://doi.org/10.1016/j.sbspro.2017.02.086, Procedia-Social and Behavioral Sciences, 237, 432-437 (2017)

Moreno, J., Reflexión en torno a la perspectiva del currículo y las aulas virtuales, Revista Inclusión y Desarrollo, 6(1), 151-157 (2019)

Musicco, G., Las soft skills \& coaching: motor de la universidad en Europa, Revista Universitaria Europea, (29), 115-132 (2018)

Ngang, T., Yunus, H., y Hashim, N., Soft skills integration in teaching professional training: novice teachers' perspectives, https://doi.org/10.1016/j.sbspro.2015.04.204, Procedia-Social and Behavioral Sciences, 186, 835-840 (2015)

Nickson, D., Warhurst, C., y otros 3 autores, Soft skills and employability: evidence from UK retail, https://doi.org/10.1177/0143831X11427589, Economic and Industrial Democracy, 33, (1), 65-84 (2012)

Niño-Pinilla, E., y Correa-Ocampo, D., Caracterización de las habilidades blandas en los sectores priorizados del área metropolitana de Cúcuta, Revista Interfaces, 2(2), 1-13 (2019)

Ortega, C., Febles, J., y Estrada, V., Una estrategia para la formación de competencias blandas desde edades tempranas, Revista Cubana de Educación Superior, 35(2), 35-41 (2016)

Rebele, J., y St. Pierre, E., Commentary on learning objectives for accounting education programs: the importance of soft skills and technical knowledge, https://doi.org/10.1016/j.jaccedu.2019.07.002, Journal of Accounting Education, 48, 71-79 (2019)

Robles, M., Executive perceptions of the top 10 soft skills needed in todays workplace, https://doi.org/10.1177/1080569912460400, Business Communication Quarterly, 75(4), 453-465 (2012)

Rueda, P., y Portilla, S., Formación en competencias laborales generales, desafío para la educación superior, Universidad de Investigación y Desarrollo, 15 (1), 37-44 (2019)

Ruíz, V., Habilidades para la vida: una propuesta de formación humana, Itinerario Educativo, 28(63), 61-89 (2014)

Schwab, K., y Samans, R., The future of jobs: employment, skills and workforce strategy for the fourth industrial, Revolution In World Economic Forum,1-32 (2016)

Tito, M., y Serrano, B., Desarrollo de soft skills una alternativa a la escasez de talento humano, INNOVA, Research Journal, 1(12), 59-76 (2016)

Tseng, H., Yi, X., y Yeh, H., Learning-related soft skills among online business students in higher education: grade level and managerial role differences In self-Regulation, Motivation, and Social Skill, https://doi.org/10.1016/j.chb.2018.11.035, Computers in Human Behavior, 95, 179-186 (2019)

Vera, F., Infusión de habilidades blandas en el currículo de la educación superior, Revista Akademèia, 7(1), 53-73 (2016)

Wilson-Ahlstrom, A., Yohalem, N., y otros cuatro autores., From soft skills to hard data: measuring youth program outcomes., The Forum for Youth Investment (2014)

Zhang, A., Peer assessment of soft skills and hard skills, Journal of Information Technology Education Research,11, 155-168 (2012) 
